\title{
Multispectral Laser Head for Terrain Identification and Analysis
}

\author{
Z. MierczyK, M. Zygmunt, M. KaszczuK* And M. Muzal
}

Institute of Optoelectronics, Military University of Technology, S. Kaliskiego 2, 00-908 Warsaw, Poland

\begin{abstract}
The reflectance profilometer, constructed in Institute of Optoelectronics, Military University of Technology, is an optoelectronic device based on the laser remote sensing technique, designed for use on unmanned aerial vehicles. The main task of the profilometer is to analyze the reflectance signals for laser radiation from the multispectral range: 850,905 , and $1550 \mathrm{~nm}$. On the basis of the analysis the profilometer enables two fundamental functions: to define the shape of the ground and identify elements of terrain coverage, along with analysis of its physicochemical properties. The reflectance profilometer is a system based on a modern concept of measurement, allowing to obtain unique information about tested objects, whose measurements or detection are not possible by means of thermal imaging systems and systems operating in the visible range. The received information is visualized in 3D format, which allows for more accurate, precise and comprehensive way of analyzed area model presentation.
\end{abstract}

DOI: 10.12693/APhysPolA.124.502

PACS: 05.70.Ce, 65.40.G-, 42.62.-b

\section{Introduction}

Reflectance profilometer is an optoelectronic device based on laser remote sensing technique. Its fundamental function is to define the shape of terrain along with the identification and analysis of coverage elements, characterized by high spatial resolution. The developed head is designed for use on unmanned aerial vehicles (UAV), Fig. 1.

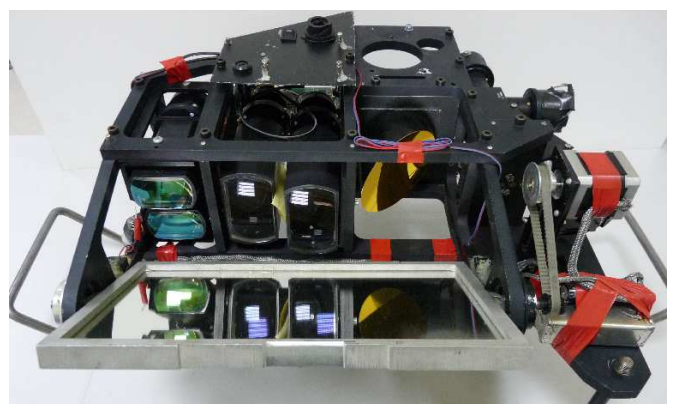

Fig. 1. Picture of the reflectance profilometer head.

Specifying parameters of the reflectance profilometer operation, it moves with a constant velocity $(100 \mathrm{~km} / \mathrm{h})$ on a fixed height $(120 \mathrm{~m})$ and through the scanning system examines a strip of land of a fixed width $(231 \mathrm{~m})$ and length (minimum $1 \mathrm{~km}$ ). Defined above parameters result directly from the requirements concerning the system design in the field of laser beams divergences, angles of view of optical detection systems or scanning speed. The sources of radiation are semiconductor pulsed laser diodes of high power. Conducted analysis of available

\footnotetext{
*corresponding author; e-mail: mszopa@wat.edu.pl
}

detector elements and the possibilities in construction of optical systems design allowed for the conclusion that in these measurement parameters the optimal diameter of the laser spot is determined by the value of approximately $0.7 \mathrm{~m}$. This value provides both the effect of averaging, as well as guarantees the possibility of effective echo signal focusing in the area of detector.

\section{Profilometer operation principles}

The basis of the profilometer operation principles is the rangefinding technique. In this technique the subject of measurements is a time interval between laser impulse emission and the moment of detection of the optical echo, which is the result of laser beam reflection from an object [1-3]. Placed at a specified height and making series of measurements of distances to a fixed grid of points distributed on the ground surface (Fig. 2), it is possible to determine a shape (profile) of analyzed terrain. In this way a digital elevation model (DEM) can be determined.

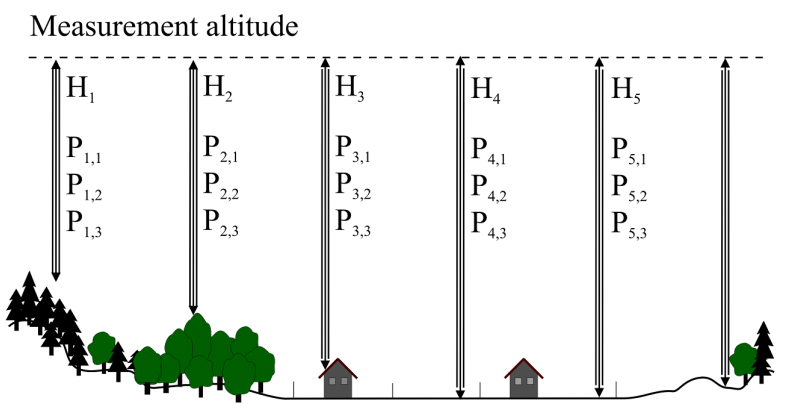

Fig. 2. Diagram of the idea of multispectral rangefinding technique.

The use in the developed laser head of three selected wavelengths of electromagnetic radiation $(850 \mathrm{~nm}$, 
$905 \mathrm{~nm}, 1550 \mathrm{~nm}$ ), leads to the introduction of basic rangefinding technique in the area of multispectral detection. Thanks to the multiplicity of number of analyzed wavelengths it is possible not only to measure a distance from objects, but also to make a comparative analysis of the intensity of optical echo signals for different wavelengths and to estimate their relationships. These relationships stem directly from spectral characteristics of analyzed materials and being their unique feature provide the basis for the process of substances identification.

\section{Identification and analysis of land cover elements}

A necessary tool on which the object identification process is based, is a database of the substances reflectance characteristics. The process of developing the database required of collection, preparation, measurements, and analysis of spectra of main types of substances and materials which could potentially be an element of a terrain cover. The measurements were conducted with the use of spectrometer Lambda 900 (Perkin Elmer), equipped with the integration sphere Pela 1001 (Fig. 3), designed for measurements of total reflectance, diffuse reflectance, and values of specular reflection [4].

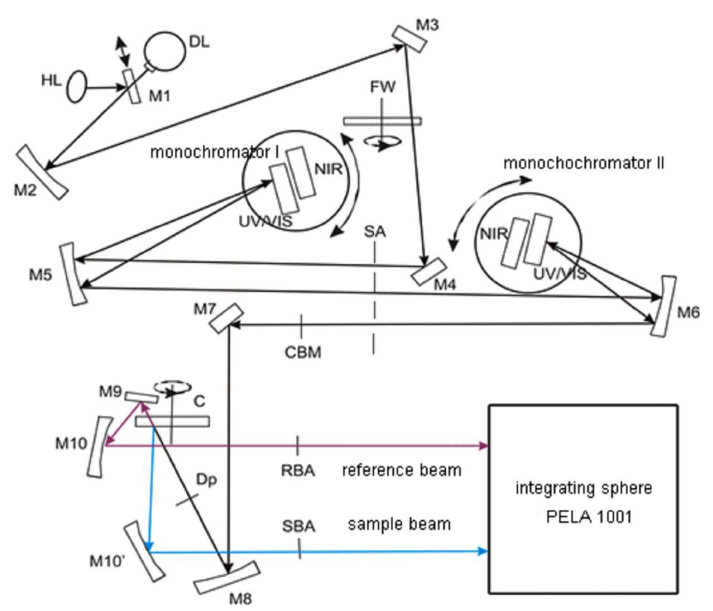

Fig. 3. Diagram of Lambda 900 spectrometer (Perkin Elmer).

The research material was chosen so as to include various kinds of substances of a different composition and complexity of construction, taking into account both inanimate substances, including natural and anthropogenic materials, as well as elements of living matter. The conducted measurements were further enriched with consideration in the measurements of various factors which may potentially bring changes in the reflectance values of analyzed materials.

Received results in the form of reflectance spectra of various media (Fig. 4) were collected and sorted. One analyzed in detail the reflectance values for three selected wavelengths: $\lambda_{1}=850 \mathrm{~nm}, \lambda_{2}=905 \mathrm{~nm}, \lambda_{3}=$

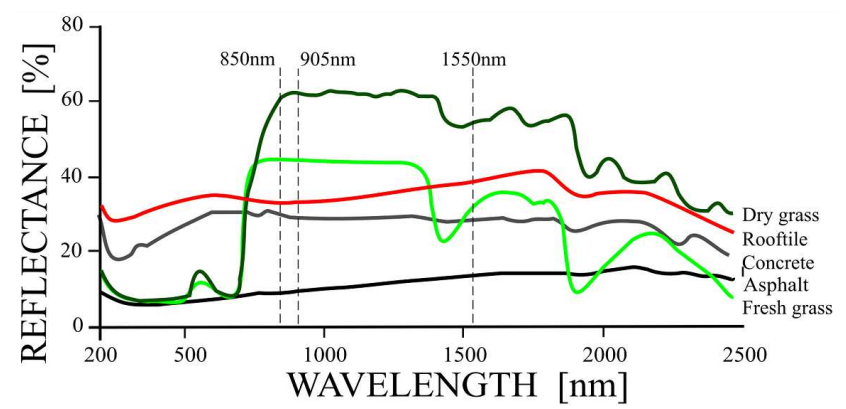

Fig. 4. Sample spectra of selected materials.

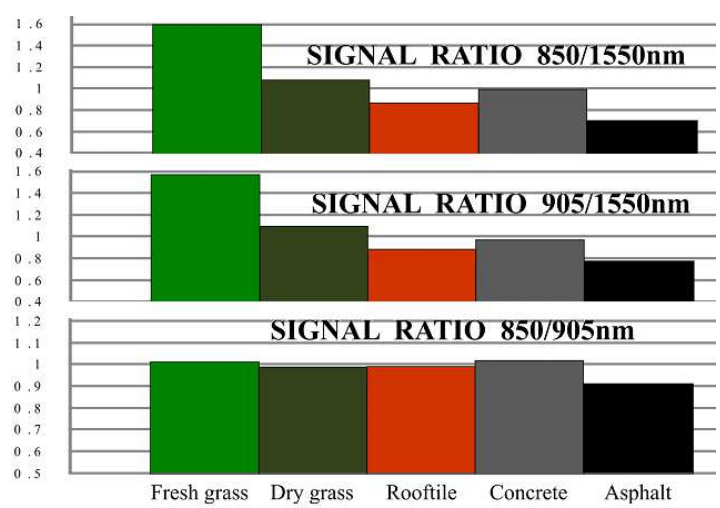

Fig. 5. Graph of reflectance signal ratios for selected materials.

$1550 \mathrm{~nm}$, and then calculated the values of signal ratios: $850 / 1550 \mathrm{~nm}, 850 / 905 \mathrm{~nm}, 905 / 1550 \mathrm{~nm}$. The values of signal ratios for different materials (Fig. 5) became a basis for the process of identification and analysis of the surface cover elements and their physico-chemical properties. Thus, the determination of digital surface model (DSM) by the laser scanner system became possible.

A sample DSM map obtained during one of the measurement campaigns is presented in Fig. 6. This map shows the area presented in satellite image (Fig. 7) derived from the resources of google maps. In the picture we can see areas of different types of land covers (asphalt, concrete, rooftiles, plants - both fresh and dry ones).

The DSM map was created by analyzing the areas of various reflectance characteristics, which were measured by the profilometer, and then assign them to the specific type of land cover material, on the basis of information gathered in the reflectance characteristics database.

The way of assigning specific material type to data obtained from the reflectance profilometer is schematically presented in Fig. 8.

\section{Summary}

The reflectance profilometer developed in the Institute of Optoelectronics, Military University of Technology is an optoelectronic device, based on the laser remote sensing technique. Its fundamental function is to determine 


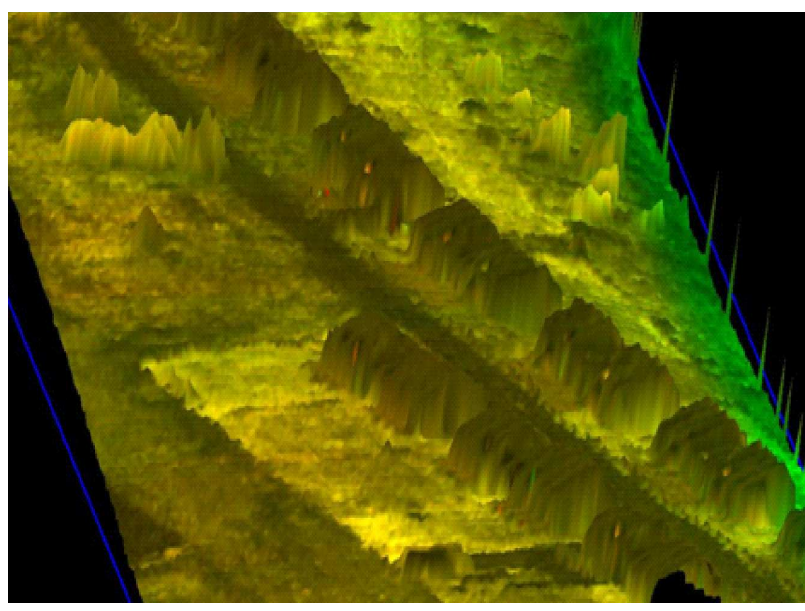

Fig. 6. Sample DSM map obtained by the reflectance profilometer.

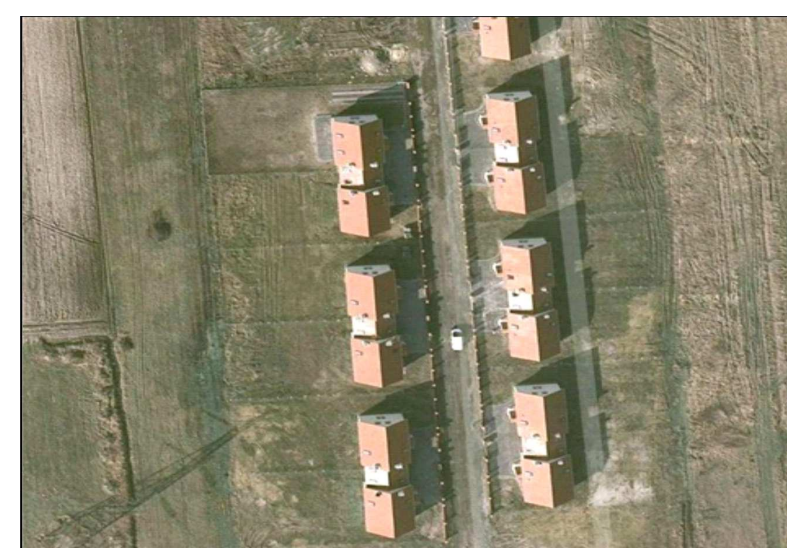

Fig. 7. Satellite image of analyzed area (resources of google maps).

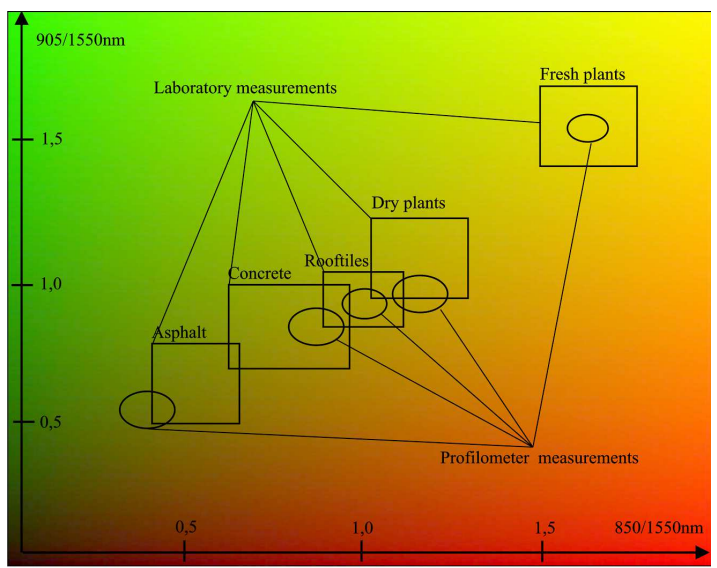

Fig. 8. Diagram of the way of assigning specific material type to data obtained from the reflectance profilometer. a shape of terrain along with the identification and analysis of land cover elements. The process of area shape determination is realized on the basis of rangefinding measurements. The land cover elements identification is possible due to the analysis of various material reflectance characteristics, which were worked out with the use of Lambda 900 spectrometer (Perkin Elmer).

The profilometer laser head operates in the range of 850-1550 nm, enabling to get unique information about tested objects, whose measurements or detection are not possible by means of thermal imaging systems and systems operating in the visible range. Imaging of obtained information in 3D format allows for more accurate and comprehensive way of presentation of an analyzed area along with physico-chemical properties of a tested surface.

\section{Acknowledgments}

The project was financed by the Polish Ministry of Science and Education under the project O R00 003708.

\section{References}

[1] C.S. Fox, The Infrared and Electro-Optical Systems Handbook, Infrared Information Analysis Center and SPIE Optical Engineering Press, Belingham, Washington 1993.

[2] T.A. Warner, M.D. Nellis, G.M. Foody, The SAGE Handbook of Remote Sensing, Sage Publications, London 2009.

[3] W.G. Rees, Physical Principles of Remote Sensing, Cambridge University Press, Cambridge 2001.

[4] M. Kaszczuk, Z. Mierczyk, M. Zygmunt, W. Piotrowski, J. Mierczyk, Proc. SPIE 8040, 80400S (2011). 